\title{
The subfamily Pteromalinae (Chalcidoidea: Pteromalidae) in South-Eastern Iran
}

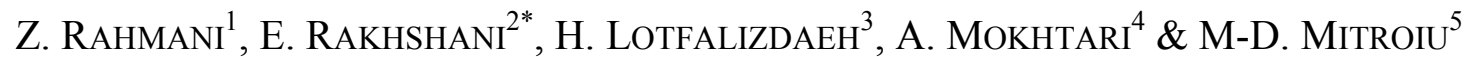 \\ ${ }^{1}$ Zahra Rahmani, Department of Plant Protection, College of Agriculture, University of Zabol, 98615-538, I. R. Iran. \\ ${ }^{2}$ Ehsan Rakhshani, Department of Plant Protection, College of Agriculture, University of Zabol, 98615-538, I. R. Iran. \\ E-mail: rakhshani@uoz.ac.ir, *Corresponding author \\ ${ }^{3}$ Hossein Lotfalizdaeh, Plant Protection Research Department, East Azarbaijan Agricultural and Natural \\ Resources Research \& Education Centre, AREEO, Tabriz, Iran. \\ ${ }^{4}$ Azizollah Mokhtari, Department of Plant Protection, College of Agriculture, University of Zabol, 98615-538, I. R. Iran. \\ ${ }^{5}$ Mircea-Dan Mitroiu, Faculty of Biology, Alexandru Ioan Cuza University Iasi Invertebrate Diversity \& Phylogenetics \\ Research Group Bd. Carol I 20A, 700505 Iasi, Romania.
}

\begin{abstract}
A faunistic survey on the parasitic wasps of the subfamily Pteromalinae Dalman, 1820 (Hym., Chalcidoidea, Pteromalidae) of South-Eastern Iran (Kerman province) was done. In total 46 specimens representing 10 genera and 16 species are identified, among them, Dinarmus altifrons (Walker, 1862) and Syntomopus incurvus Walker, 1833 are recorded for the first time for the fauna of Iran. Previously recorded species of Pteromalinae from this area are also reviewed and listed. Faunistic analysis of the known species according to their distribution both in Iran and in a broader biogeographical sense is also discussed.
\end{abstract}

Keywords. Parasitoids, Iran, fauna, new records, distribution, map.

\section{INTRODUCTION}

$\mathrm{P}$ teromalidae is one of the most diverse families of the parasitic wasps with a worldwide distribution, comprising 33 subfamilies with a wide variety of taxonomic complications (Noyes 2019). About $98 \%$ of Pteromalidae are recognised as parasitoids on various stages of insects of many orders or rarely, of spiders, therefore they play a significant role in the control of many pests in agriculture, forestry, and natural areas (Bouček \& Rasplus 1991). The subfamily Pteromalinae Dalman, 1820 is the most species-rich group within Pteromalidae with more than 2330 described species in over 317 genera, distributed worldwide (Noyes 2019). Still, very little is known about the Pteromalinae fauna in Iran. Lotfalizadeh \& Gharali (2008) provided the first checklist of Iranian Pteromalidae, which includes 78 species from various regions.
The recently published checklist of Pteromalidae (Hymenoptera: Chalcidoidea) of Iran (Abolhassanzadeh et al. 2017) includes 62 genera and 129 species, of which 44 genera and 91 species belong to the subfamily Pteromalinae. Further Pteromalinae species have recently been found by the subsequent research (Moravvej et al. 2018, Lotfalizadeh et al. 2020, Rahmani et al. 2019, 2020, Shojaey et al. 2019, 2021, Gibson et al. 2021) bringing the number of known Iranian species of Pteromalinae to 113 , and this also indicated the least contribution on this diverse group of parasitoids. Among the explored areas in South-Eastern parts of Iran, a moderately good knowledge of Pteromalinae is already devoted to Kerman province (Mitroiu et al. 2011, Lotfalizadeh et al. 2012, Mahdavi \& Madjdzadeh 2013, Ziaaddini et al. 2014, Mahdavi et al. 2015, Rahmani et al. 2019, Shojaey et al. 2019, 2021), the areas contributed as the largest province of Iran with a 
rather wide variety of climatic and habitat diversity. Our recent infrequent surveys in these areas led to the discovery of additional Pteromalinae species, presented in this paper.

\section{MATERIAL AND METHODS}

The material for the present study was sporadically collected in various regions of the South-Eastern part of Iran (Kerman province) by Malaise traps and using a sweep net, during 2013-2019. The specimens were treated and prepared according to the AXA protocol (van Achterberg 2009), then card mounted and labelled. The external morphology of the specimens was studied under a Nikon ${ }^{B}$ SMZ645 stereomicroscope and photographed using a Canon ${ }^{\circledR}$ EOS 700D (Canon ${ }^{\circledR}$ Inc., Japan) camera, mounted with an adapter on Hund $^{\circledR}$ Stereomicroscope (Wetzlar Inc., Germany). Identification of the specimens was done based on the relevant keys and descriptions (Graham 1969, Sureshan \& Narendran 2001, Dzhanokmen 2009, Mitroiu 2012). The terminology of the morphological characters generally follows that of Graham (1969) and Gibson et al. (1997). Data about the distribution of species are mainly extracted from Noyes (2019). The studied specimens are deposited in the collection of the Department of Plant Protection, University of Zabol, Iran (DPPZ). Previously recorded species of Pteromalinae from Kerman province were also listed.

The following abbreviations have been used for some morphological terms: POL for posterior ocellar distance; OOL for ocello-ocular distance.

The institutes, where the type specimens were deposited are as follows: BMNH - The Natural History Museum, London, England; CUTT Plant Protection Department, Cumhuriyet Universitesi, Tokat Ziraat Fakültesi, Tokat, Turkey; LUZN - Zoological Museum, Lund University, Sweden; MLSF - Museum "La Specola", Florence, Italy; NHRM - Naturhistoriska Riksmuseet, Stockholm, Sweden; QMB - Queensland Museum, Brisbane, Australia, and ZMUC - Zoologiske Museum, Copenhagen, Denmark.

\section{RESULTS}

In total, 16 species belonging to 10 genera of the subfamily Pteromalinae are listed, among them, four genera and eight species are first records for the fauna of Kerman province. Dinarmus altifrons (Walker, 1862) and Syntomopus incurvus Walker, 1833 are also recorded for the first time from Iran. Newly recorded species are marked with an asterisk $\left(^{*}\right)$.

\section{Family Pteromalidae Dalman, 1820}

\section{Subfamily Pteromalinae Dalman, 1820}

\section{Genus Caenocrepis Thomson, 1878}

Caenocrepis Thomson, 1878: 51.

Type species: Dimachus arenicola Thomson, 1878 , by monotypy.

\section{Caenocrepis arenicola (Thomson, 1878)}

Dimachus arenicola Thomson, 1878: 51, Lectotype $q$. - LUZN, Sweden.

Material examined. 19 , Iran, Kerman province, Jiroft (2840'31.17"N, 5744'16.69"E, 686 m), swept on Mentha pulegium, 04.09.2014, N. Amirinasab, leg.

Distribution in Iran. Kerman province (Shojaey et al. 2019, 2021, current study).

General distribution. Eastern Palaearctic (Iran - Shojaey et al. 2019, Kazakhstan), Western Palaearctic (Europe, Morocco, Turkey).

\section{Genus Cheiropachus Westwood, 1829}

Cheiropachus Westwood, 1829: 23.

Type species: Ichneumon quadrum Fabricius, 1787 , by original designation.

\section{Cheiropachus quadrum (Fabricius, 1787)}

Ichneumon quadrum Fabricius, 1787: 270, 今̂. - ZM $\mathrm{UC}$, Germany. 
Material examined. 899 , Iran, Kerman province: Baft $\left(29^{\circ} 22^{\prime} 35.96^{\prime \prime} \mathrm{N}, 56^{\circ} 40^{\prime} 18.22\right.$ "E, 2736 $\mathrm{m})$, Malaise trap, 11.05.2018, 3ㅇ; Golbaf $\left(29^{\circ} 52^{\prime} 54.53^{\prime \prime} \mathrm{N}, 57^{\circ} 43^{\prime} 40.71 " \mathrm{E}, 1743 \mathrm{~m}\right)$, Malaise trap, 10.04.2018, 5우, Sh. Mohebban, leg.

Distribution in Iran. Ardabil (Basiri et al. 2012), East Azarbaijan (Lotfalizadeh \& Gharali 2008), and Kerman (Mitroiu et al. 2011, current study) provinces.

General distribution. Eastern Palaearctic (China, Iran, Kazakhstan, Kyrgyzstan, Pakistan, Turkmenistan), Nearctic (Canada, U.S.A), Neotropical (Argentina, Chile), Oriental (India), Western Palaearctic (Armenia, Europe, Caucasus, Egypt, Israel, Lebanon, Morocco, North Africa, Russia, Tunisia, Turkey).

\section{Genus: Dinarmus Thomson, 1878}

Dinarmus Thomson, 1878: 56 (as subgenus of Dimachus Thomson).

Type species: Dimachus acutus Thomson, 1878. Designated by Ashmead 1904.

\section{Dinarmus altifrons (Walker, 1862)*}

(Figures 1-2)

Pteromalus altifrons Walker, 1862: 388, Lectotype + . - BMNH, South Africa.

Material examined. $6 ㅇ+$, 2 $\hat{\jmath}$, Iran, Kerman province, Bardsir $\left(29^{\circ} 56^{\prime} 20.01^{\prime \prime N}, 5^{\circ} 34^{\prime} 41.84 " \mathrm{E}\right.$, 2040 m): swept on Sophora sp., 12.08.2013, 3 우; swept on Glycyrrhiza glabra, 06.08.2013,

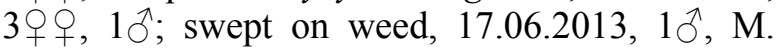
Azad-Biglari, leg.

Diagnosis. Female (Figs 1-2A). Body length: $2.8-3.0 \mathrm{~mm}$. Head in frontal view $1.24-1.25$ times wider than high; (Fig. 1A). Clypeal margin bidentate (Fig. 1A). Toruli inserted above lower margins of eyes (Fig. 1A). Antenna with scape reaching lower edge of median ocellus (Fig. 1A). Width of head 2.7-2.9 times its median length (dorsal view) (Fig. 1B). POL 2-2.14 times longer than OOL (Fig. 1B). Eye height 1.54-1.64 times longer than its length (lateral view) (Fig. 1C). Mesoscutum 1.6-1.8 times wider than its length (Fig. 1D). Fore wing with basal cell and basal fold glabrous, marginal, postmarginal and stigmal veins almost equal in length, stigma large (Fig. 1E). Propodeum coarsely reticulate, median carina and costula absent; plicae weakly indicated (Fig. 1F). Gaster sessile, ovate, elongate. Body metallic green with bronze-copper reflection, wings hyaline, veins yellow, setae pale yellow and stigma dark brown (Fig. 2A).

Male (Fig. 2B). Body length: 2-2.3 mm. Similar to female, but antenna with two anelli and six funicular segments, costal cell forewing widened with dense bristles, marginal vein shorter and wider, body metallic green with weak bronzecopper reflection, wings hyaline, veins and setae brown.

Distribution in Iran. Kerman province (current study).

General distribution. Afrotropical (South Africa), Oriental (India), Eastern (Pakistan, Iran [New record]) and Western (Algeria, Israel) Palaearctic.

\section{Genus: Euneura Walker, 1844}

Euneura Walker, 1844: 331.

Type species: Euneura augarus Walker, 1844, by monotypy.

\section{Euneura lachni (Ashmead, 1887)}

Pachycrepis lachni Ashmead, 1887: 193.

Material examined. 590 , Iran, Kerman province: Rayen $\left(29^{\circ} 35^{\prime} 51.22^{\prime \prime} \mathrm{N}, \quad 57^{\circ} 28^{\prime} 03.04 " \mathrm{E}\right.$, $2093 \mathrm{~m}$ ), Malaise trap, 25.05.2018, 3우; Rabor $\left(29^{\circ} 18^{\prime} 11.39^{\prime \prime} \mathrm{N}, 56^{\circ} 52^{\prime} 53.28^{\prime \prime} \mathrm{E}, 2440 \mathrm{~m}\right)$, Malaise trap, 20.06.2018, 2ㅇ, Sh. Mohebban, leg.

Distribution in Iran. Ardabil, Hamadan, Tehran (Rakhshani et al. 2005), and Kerman (current study) provinces; No exact locality cited (OILB 1971). 

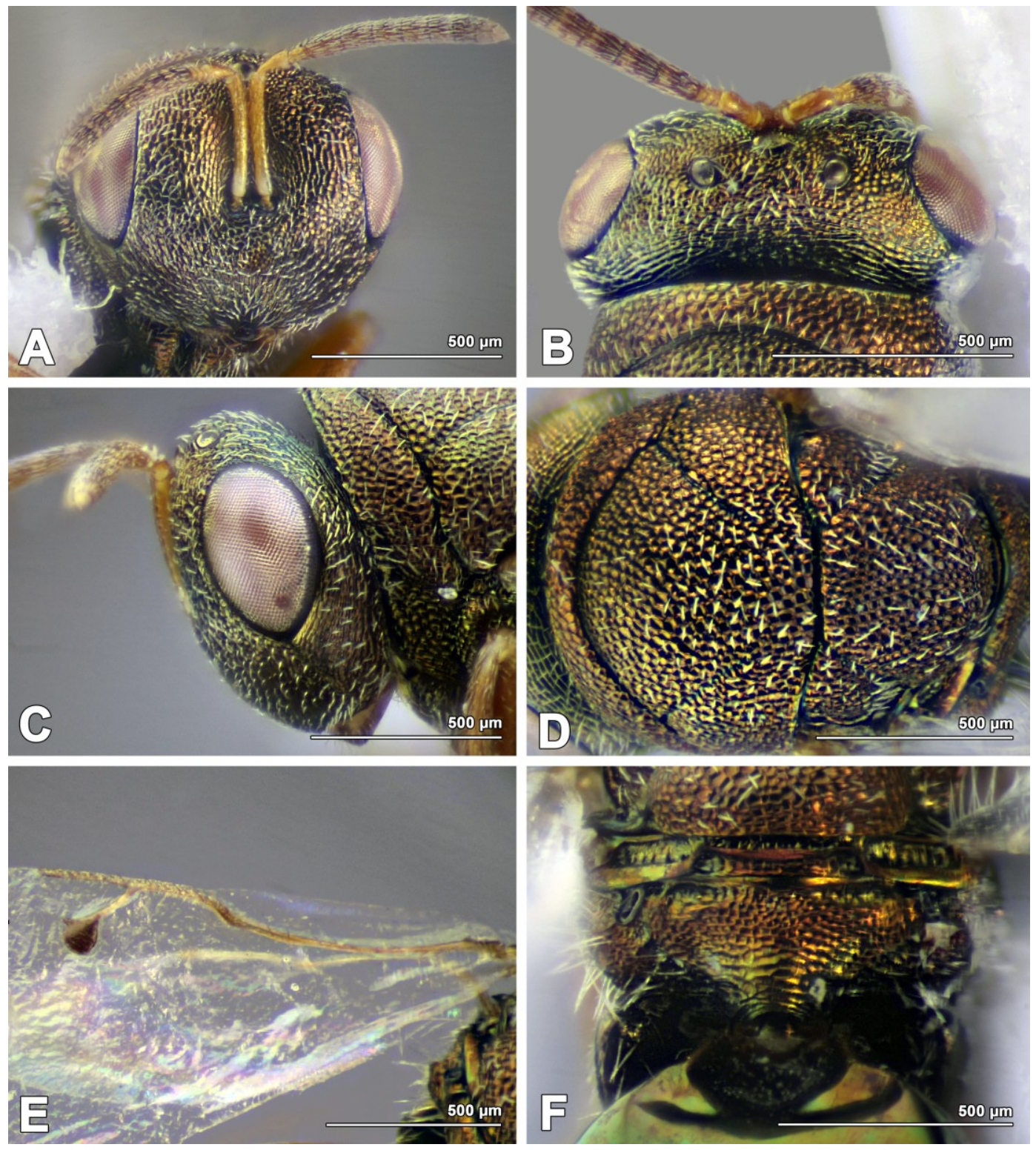

Figure 1. Dinarmus altifrons (Walker, 1862). Female. $\mathrm{A}=$ Head in frontal view, $\mathrm{B}=$ Head in dorsal view, $\mathrm{C}=$ Head in lateral view, $\mathrm{D}=$ Mesosoma in dorsal view, $\mathrm{E}=$ Fore wing venation, $\mathrm{F}=$ Propodeum in dorsal view .

General distribution. Eastern Palaearctic (Iran, Japan, Kyrgyzstan, Korea, Pakistan, China), Nearctic (Canada, USA), Oriental (India), Western Palaearctic (Europe, Russia).

\section{Genus Habritys Thomson, 1878}

Habritys Thomson, 1878: 54 (as subgenus of Dimachus Thomson).
Type species: Pteromalus brevicornis Ratzeburg 1844, by monotypy.

\section{Habritys brevicornis (Ratzeburg, 1844)}

Pteromalus brevicornis Ratzeburg, 1844: 201.

Material examined. 1 , , Iran, Kerman province, Bardsir $\left(29^{\circ} 52^{\prime} 04.69^{\prime \prime N}, 56^{\circ} 38^{\prime} 53.36^{\prime \prime} \mathrm{E}\right.$, 


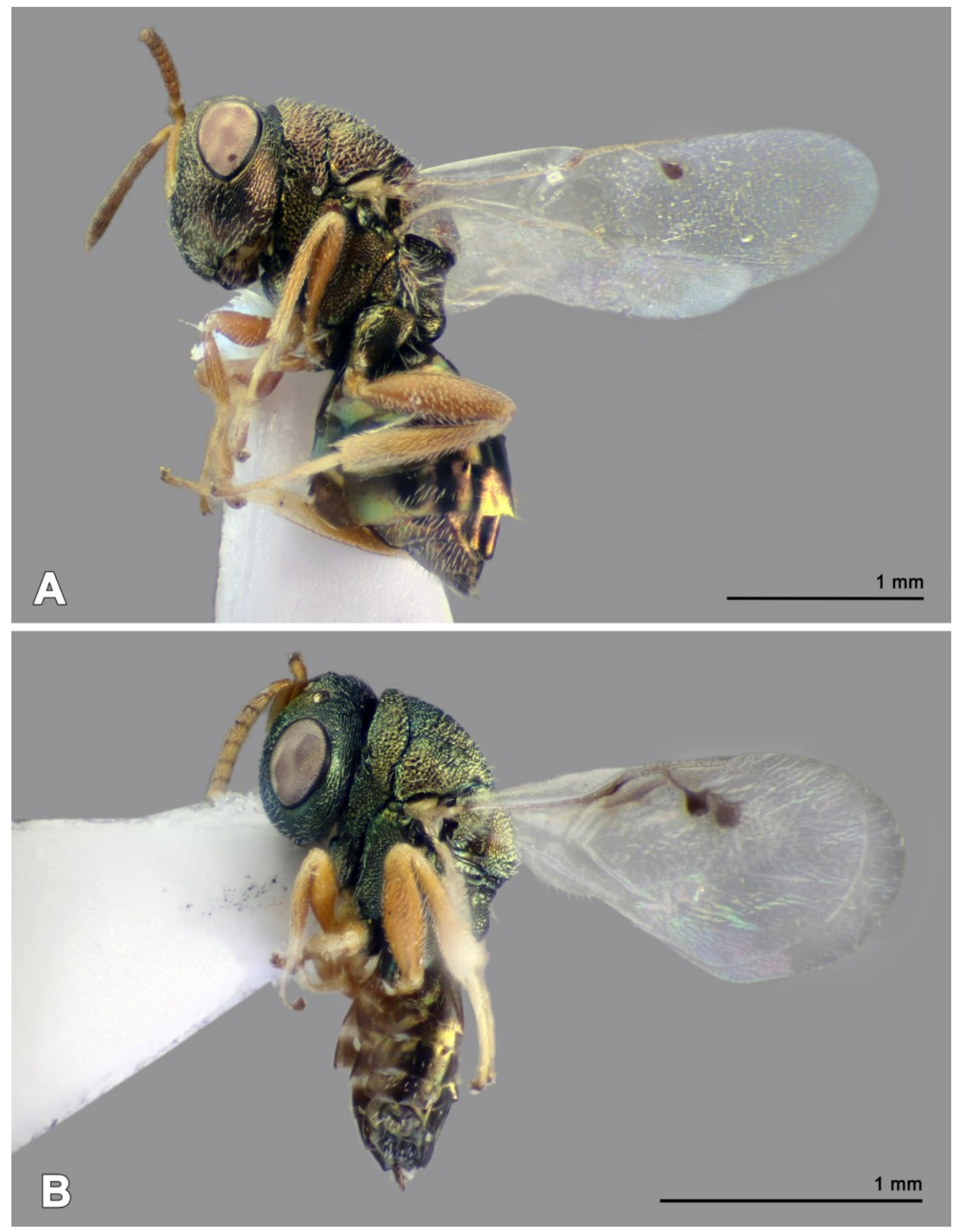

Figure 2. Dinarmus altifrons (Walker, 1862). Lateral view of habitus, $\mathrm{A}=$ Female, $\mathrm{B}=$ Male

$2088 \mathrm{~m}$ ), Malaise trap, 03.05.2018, Sh. Mohebban, leg.

Distribution in Iran. East Azarbaijan, West Azarbaijan (Hassan-Pashaimehr \& Lotfalizadeh 2015), and Kerman (current study) provinces.

General distribution. Eastern Palaearctic (Iran Hassan-Pashaimehr \& Lotfalizadeh 2015, Ka- zakhstan), Nearctic (Canada, U.S.A), Western Palaearctic (Europe).

\section{Genus: Homoporus Thomson, 1878}

Homoporus Thomson, 1878: 60, 64.

Type species: Pteromalus fulviventris Walker, 1835. Designation by Ashmead, 1904. 


\section{Homoporus apharetus (Walker, 1839)}

Pteromalus apharetus Walker, 1839: 228, Lectotype ๙. - BMNH, United Kingdom.

Material examined. 19 , Iran, Kerman province, Koohpayeh (30²8'50.65"N, 57¹9'17.98" E, $1803 \mathrm{~m}$ ), Malaise trap, 28.06.2018, Sh. Mohebban, leg.

Distribution in Iran. Kerman province (Shojaey et al. 2021, current study).

General distribution. Eastern (China, Iran, Kazakhstan, Kyrgyzstan, Russia) and Western (Europe) Palaearctic.

\section{Genus Pachycrepoideus Ashmead, 1904}

Pachycrepoideus Ashmead, 1904: 329.

Type species: Pachycrepoideus dubius Ashmead, 1904, by monotypy.

\section{Pachycrepoideus vindemmiae (Rondani, 1875)}

Pteromalus vindemmiae Rondani, 1875: 145-148, Lectotype 9 . - MLSF, Italy.

Material examined. 19, Iran, Kerman province, Jiroft (28 $33^{\prime} 16.99^{\prime \prime} \mathrm{N}, 57^{\circ} 43^{\prime} 38.84^{\prime \prime} \mathrm{E}, 602$ $\mathrm{m})$, Malaise trap, 10.05.2019, Sh. Mohebban, leg.

Distribution in Iran. Kerman (Shojaey et al. 2021, current study), and Qom (Farahani et al. 2010) provinces.

General distribution. Cosmopolitan.

\section{Genus: Pachyneuron Walker, 1833}

Pachyneuron Walker, 1833: 371, 380.

Type species: Pachyneuron formosum Walker, 1833 , by monotypy.

\section{Pachyneuron aphidis (Bouché, 1834)}

Diplolepis aphidis Bouché, 1834: 170, ㅇ․ - Germany.
Material examined. 290 , Iran, Kerman province, Bardsir $\left(29^{\circ} 56^{\prime} 20.01^{\prime \prime N}, 56^{\circ} 34^{\prime} 41.84^{\prime \prime} \mathrm{E}\right.$, $2040 \mathrm{~m}$ ), swept on Weed, 30.05.2013, M. AzadBiglari, leg.

Distribution in Iran. Ardabil (Lotfalizadeh \& Gharali 2008), Kerman (Mitroiu et al. 2011, Shojaey et al. 2021, current study), Khorasan-e Razavi (Hasani \& Madjdzadeh 2012), Khuzestan (Moravvej et al. 2018), and Tehran (Rakhshani et al. 2004) provinces.

General distribution. Cosmopolitan.

Pachyneuron erzurumicum Doğanlar, 1986

Pachyneuron erzurumicum Doğanlar, 1986: 25, 26-30, Holotype 9 . - CUTT, Turkey.

Material examined. $5 q \propto$; Iran, Kerman province, Rayen $\left(29^{\circ} 35^{\prime} 51.22^{\prime \prime} \mathrm{N}, \quad 57^{\circ} 28^{\prime} 03.04^{\prime \prime} \mathrm{E}\right.$, 2093 m), Malaise trap, 25.05.2018, Sh. Mohebban, leg.

Distribution in Iran. Ilam (Lotfalizadeh \& Gharali 2008), Kerman (Mitroiu et al. 2011, current study), and Khorasan-e Razavi (Hasani \& Madjdzadeh 2012) provinces.

General distribution. Eastern Palaearctic (Iran, Kazakhstan), Western Palaearctic (Turkey).

\section{Pachyneuron formosum Walker, 1833}

Pachyneuron formosum Walker, 1833: 380, Lectotype 오. - BMNH, United Kingdom.

Material examined. 19, Iran, Kerman province, Rabor $\left(29^{\circ} 18^{\prime} 11.9^{\prime \prime} \mathrm{N}, 56^{\circ} 52^{\prime} 53.28^{\prime \prime} \mathrm{E}, 2440\right.$ $\mathrm{m})$, Malaise trap, 24.06.2018, Sh. Mohebban, leg.

Distribution in Iran. Ilam (Lotfalizadeh \& Gharali 2008), Kerman (current study), and Kordestan (Dehdar \& Madjdzadeh 2016) provinces.

General distribution. Eastern Palaearctic (China, Iran, Japan, Kazakhstan, Kyrgyzstan, Tadzhik- 
istan), Western Palaearctic (Europe, Morocco, Turkey).

\section{Pachyneuron grande Thomson, 1878}

Pachyneuron grande Thomson, 1878: 29, Lectotype.+ - LUZN, Sweden.

Material examined. 1q, Iran, Kerman province, Jiroft $\left(28^{\circ} 40^{\prime} 31.17^{\prime \prime} \mathrm{N}, 57^{\circ} 44^{\prime} 16.69^{\prime \prime} \mathrm{E}, 686\right.$ $\mathrm{m})$, swept on weed, 10.04.2013, N. Amirinasab, leg.

Distribution in Iran. Kerman province (current study), and Northern coastal (Sadeghi \& Ebrahimi 2001).

General distribution. Eastern Palaearctic (China, Iran, Kazakhstan, Kyrgyzstan), Western Palaearctic (Europe).

\section{Pachyneuron groenlandicum (Holmgren, 1872)}

Pteromalus groenlandicus Holmgren, 1872: 100, Lectotype + . - NHRM, Greenland.

Material examined. 19 , Iran, Kerman province, Baft $\left(29^{\circ} 22^{\prime} 35.96^{\prime \prime} \mathrm{N}, 56^{\circ} 40^{\prime} 18.22^{\prime \prime} \mathrm{E}, 2736\right.$ $\mathrm{m})$, Malaise trap, 11.05.2018, Sh. Mohebban, leg.

Distribution in Iran. Alborz (Haeselbarth 1983), Ilam (Lotfalizadeh \& Gharali 2008), Khorasan-e Razavi (Hasani \& Madjdzadeh 2012), and Kerman (Mitroiu et al. 2011, current study) provinces.

General distribution. Afrotropical (Yemen), Oriental (India), Nearctic (Greenland), Eastern Palaearctic (China, Iran, Japan, Kazakhstan, Korea), Western Palaearctic (Europe, Turkey).

\section{Pachyneuron muscarum (Linnaeus, 1758)}

Ichneumon muscarum Linnaeus, 1758: 567, Sweden.

Material examined. 2 우, Iran, Kerman province, Koohpayeh $\left(30^{\circ} 28^{\prime} 50.65^{\prime \prime} \mathrm{N}, 5^{\circ} 19^{\prime} 17.98^{\prime \prime}\right.$
E, 1803 m), Malaise trap, 28.06.2018, Sh. Mohebban, leg.

Distribution in Iran. Alborz (Ebrahimi 2014), East Azarbaijan (Lotfalizadeh et al. 2014), Fars (Lotfalizadeh \& Ahmadi 2000 - as Pachyneuron concolor Förster, 1841, Ebrahimi 2014), Kerman (current study), Kermanshah (Jalilvand et al. 2014), and Tehran (Haeselbarth 1989 - as Pachyneuron concolor) provinces.

General distribution. Afrotropical (Saudi Arabia), Oriental (India), Neotropical (St Vincent \& Grenadines), Eastern Palaearctic (China, Iran, Kazakhstan, Taiwan), Western Palaearctic (Armenia, Europe, Caucasus, Georgia, Israel, Russia, Turkey).

\section{Pachyneuron nelsoni Girault, 1928}

Pachyneuron nelsoni Girault, 1928: 2, Holotype ㅇ. QMB, Australia-Queensland.

Material examined. $3 ㅇ+$, Iran, Kerman province, Rayen $\left(29^{\circ} 35^{\prime} 51.22^{\prime \prime N}, \quad 57^{\circ} 28^{\prime} 03.04^{\prime \prime} \mathrm{E}\right.$, $2093 \mathrm{~m}$ ), Malaise trap, 25.05.2018, Sh. Mohebban, leg.

Distribution in Iran. East Azarbaijan (Lotfalizadeh \& Gharali 2008), Kerman (Shojaey et al. 2021, current study), Khorasan-e Razavi (Hasani \& Madjdzadeh 2012), and Kordestan (Dehdar \& Madjdzadeh 2016) provinces.

General distribution. Cosmopolitan

\section{Genus: Rhaphitelus Walker, 1834}

Rhaphitelus Walker, 1834: 168, 178.

Type species: Rhaphitelus maculatus Walker, 1834 , by monotypy.

\section{Rhaphitelus maculatus Walker, 1834}

Rhaphitelus maculatus Walker, 1834: 179, Holotype 9. - BMNH, United Kingdom. 
Material examined. $2 q 9$, Iran, Kerman province: Rayen $\left(29^{\circ} 35^{\prime} 51.22^{\prime \prime} \mathrm{N}, \quad 57^{\circ} 28^{\prime} 03.04^{\prime \prime} \mathrm{E}\right.$, $2093 \mathrm{~m}$ ), Malaise trap, 27.07.2018, 19; Golbaf $\left(29^{\circ} 52^{\prime} 54.53^{\prime \prime} \mathrm{N}, 57^{\circ} 43^{\prime} 40.71^{\prime \prime E}, 1743 \mathrm{~m}\right)$, Malaise trap, 19.04.2018, 1ㅇ, Sh. Mohebban, leg.

Distribution in Iran. Alborz, Ardabil, East Azarbaijan, Hamedan, Isfahan, Markazi, Zanjan (Abolhassanzadeh et al. 2017), and Kerman (current study) provinces.

General distribution. Australian (Australia), Eastern Palaearctic (Iran, Japan, Kazakhstan, Kyrgyzstan, China, Turkmenistan, Uzbekistan), Nearctic (Canada, USA), Neotropical (Argentina, Chile), Oceanic (New Zealand), Oriental (India), Western Palaearctic (Egypt, Europe, Israel, Russia, Tunisia, Turkey).

\section{Genus: Syntomopus Walker, 1833}

Syntomopus Walker, 1833: 371, 372:

Type species: Syntomopus thoracicus Walker, 1833. Designation by Westwood 1839.

\section{Syntomopus incurvus Walker, 1833*}

(Figures 3-4)

Syntomopus incurvus Walker, 1833: 372, Lectotype 9. - BMNH, United Kingdom.

Material examined. $3+$ 우 $10^{\lambda}$, Iran, Kerman province: Koohpayeh $\left(30^{\circ} 28^{\prime} 50.65^{\prime \prime} \mathrm{N}, \quad 57^{\circ} 19^{\prime}\right.$ $17.98 " \mathrm{E}, 1803 \mathrm{~m}$ ), Malaise trap, 07.02.2018, 1 우 Fahraj (28 $\left.53^{\prime} 10.57^{\prime \prime} \mathrm{N}, \quad 58^{\circ} 47^{\prime} 22.88^{\prime \prime} \mathrm{E}, 726 \mathrm{~m}\right)$, Malaise trap, 12.06.2019, 19, 1 ${ }^{\lambda}$, Sh. Mohebban, leg.; Jiroft $\left(28^{\circ} 40^{\prime} 31.17^{\prime \prime N}, 57^{\circ} 44^{\prime} 16.69^{\prime \prime} \mathrm{E}, 686\right.$ m), swept on Mentha pulegium, 21.05.2014, 1옹 N. Amirinasab, leg.

Diagnosis. Female (Figs 3-4A). Body length: 1.9-2.25 mm. Head in frontal view 1.23-1.29 times wider than high (Fig. 3A). Toruli inserted above lower margins of eyes (Fig. 3A). Antenna with scape not reaching lower edge of median ocellus; funicular segments transverse except first segment quadrate (Fig. 3A). Width of head 2.33-
2.46 times its length (dorsal view) (Fig. 3B). POL 1.55-1.75 times longer than OOL (Fig. 3B). Eye height 1.66-1.76 times longer than its length (lateral view) (Fig. 3C). Pronotum with lateral angles almost prominent. Mesoscutum 1.8-1.84 times wider than its length (Fig. 3D). Fore wing with basal cell and basal fold almost glabrous except few setae on basal fold, marginal vein 1.2 1.23 times longer than postmarginal vein and 2.36-2.4 times longer than stigmal vein (Fig. 3E). Propodeum with median area coarsely reticulate, lateral parts smooth, median carina complete, plicae distinct (Fig. 3F). Gaster 1.61-1.65 times longer than wide; petiole 1.8-1.9 times longer than wide and with parallel sides; posterior margin of first tergite emarginated. Head and mesosoma dark green with metallic yellow reflection; Antenna dark brown with scape yellow; Legs with coxa concolorous with mesosoma, remainder segments yellow. Wings hyaline, venation light brown; metasoma dark brown with petiole black (Fig. 4A).

Male (Fig. 4B). Body length: $1.55-1.75 \mathrm{~mm}$. Similar to female, but antenna with funicular segments slightly longer than width, decreasing in length towards apex; marginal vein 2.1-2.12 times longer than stigmal vein.

Distribution in Iran. Kerman province (current study).

General distribution. Eastern (Iran [New record], Kazakhstan, China) and Western (Europe, Turkey) Palaearctic.

\section{DISCUSSION}

On the basis of the new findings, four genera (Euneura Walker, Habritys Thomson, Rhaphitelus Walker and Syntomopus Walker) and eight species of Pteromalinae (Dinarmus altifrons, Euneura lachni, Habritys brevicornis, Pachyneuron formosum, Pachyneuron grande, Pachyneuron muscarum, Rhaphitelus maculatus, and Syntomopus incurvus) are first recorded for Kerman province. Considering the habitat diversity in Kerman province as the largest province of Iran, many more species than the listed taxa (Table 1) 

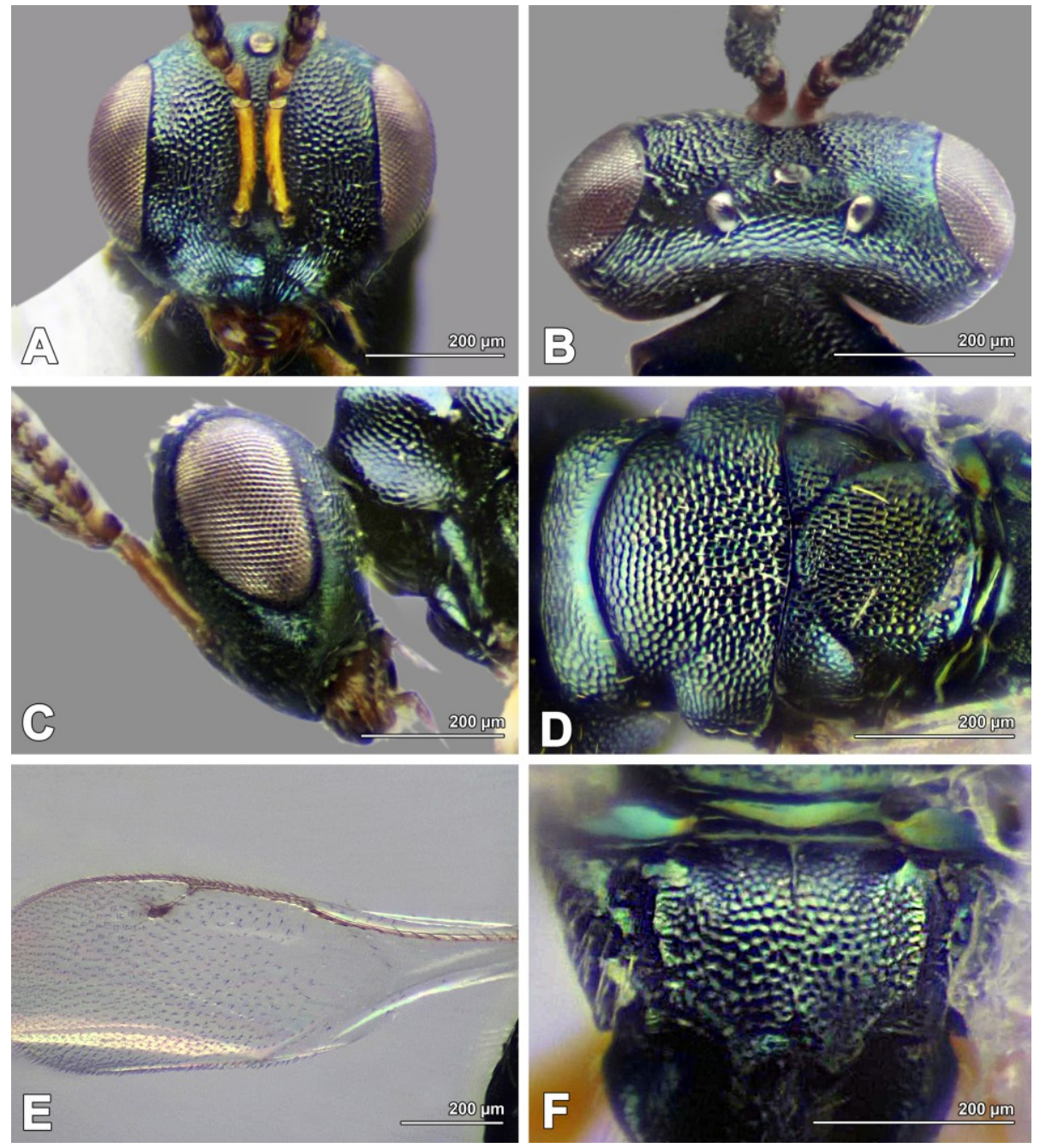

Figure 3. Syntomopus incurvus Walker, 1833. Female. $\mathrm{A}=$ Head in frontal view, $\mathrm{B}=$ Head in dorsal view, $\mathrm{C}=\mathrm{Head}$ in lateral view, $\mathrm{D}=$ Mesonotum in dorsal view, $\mathrm{E}=$ Fore wing venation, $\mathrm{F}=$ Propodeum in dorsal view.

are expected. On the other hand, very little is known about the occurrence of the known species in other provinces of Iran (Figure 5). The 45 species of Pteromalinae recorded in Kerman comprise only $39.8 \%$ of the known Pteromalinae from Iran (Abolhassanzadeh et al. 2017, Moravvej et al. 2018, Lotfalizadeh et al. 2020, Rahmani et al. 2019, 2020, Shojaey et al. 2019, 2021, Gibson et al. 2021). Two species, Dinarmus altifrons and Syntomopus incurvus are also representing new records for the fauna of Iran. Three Dinarmus species (Eslami 1998, Hasani et al. 2011, Mitroiu et al. 2011) and only a single Syntomopus species (Lotfalizadeh \& Gharali 2008) were already re- corded from Iran. The genera Dinarmus and Syntomopus have seven and nine species in the Palaearctic region, respectively (Noyes 2019).

The Iranian specimens of Dinarmus altifrons were rather similar to the description of the lectotype from South Africa (Rasplus 1989). The marginal clypeal dentacles make a V-shape incision, a character that is slightly different from the U-shape incision illustrated in the African specimens and more similar to the Indian specimens of D. altifrons (Sureshan \& Narendran 2001). The scape reaches the lower margin of the median ocellus and the propodeal nucha sculpture 


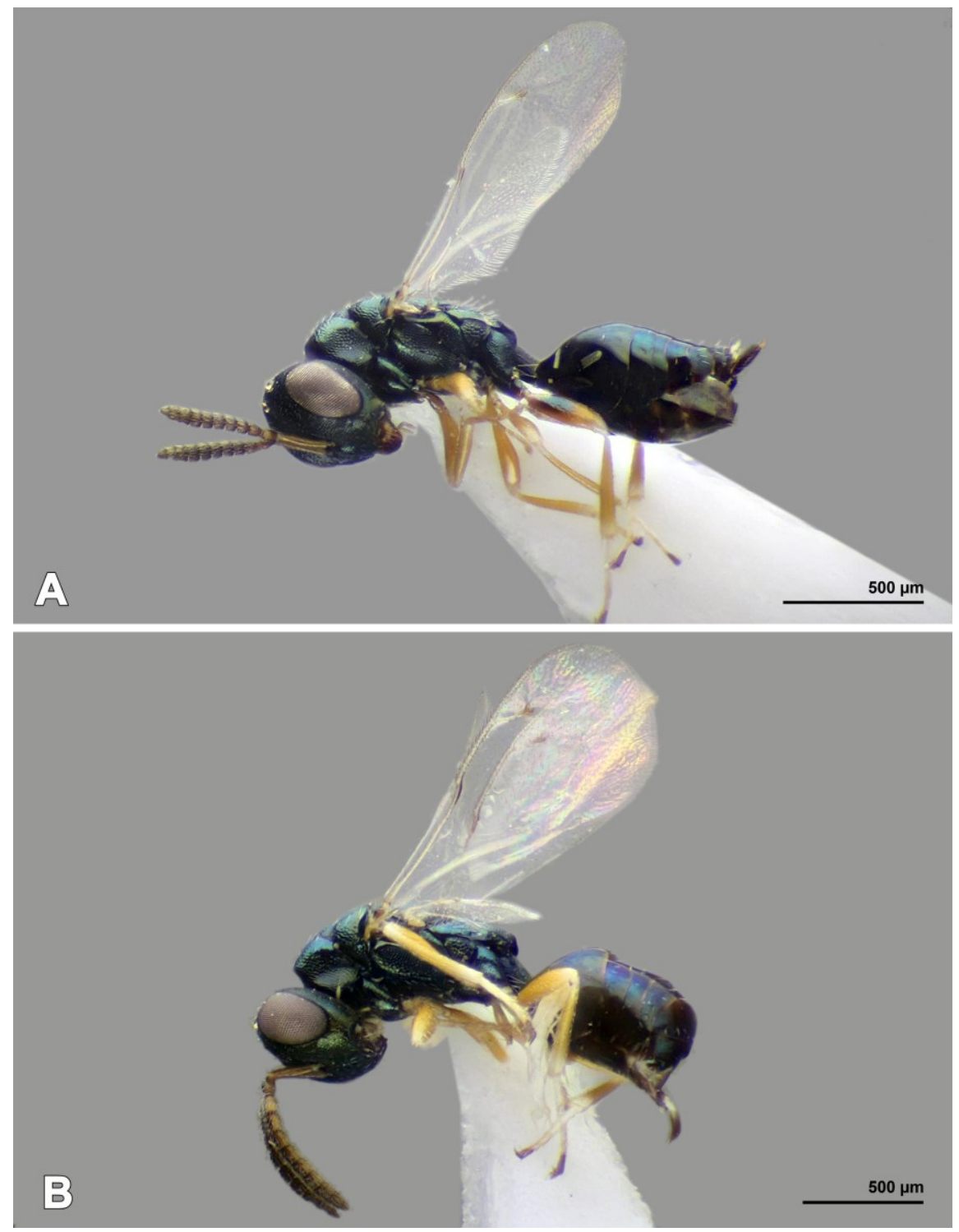

Figure 4. Syntomopus incurvus Walker, 1833 - Lateral view of habitus. A. Female; B. Male.

is a bit different. Considering the type locality, and the few minor differences, the specimens outside South Africa may represent a different species. This should be verified by a direct examination of the type specimens (BMNH London), and also by assessing the intraspecific variability through examination of many specimens from various regions. A world revision of the genus or at least of the species group would also be necessary to include molecular data.
The majority of the known Pteromalinae species in Kerman (42.2\%) are not recorded outside the Palaearctic region. On the other hand, $20 \%$ of species share a distribution both in the Palaearctic and the Nearctic regions (Table 2). Not enough data are yet gathered to make an appropriate judgment about such distribution unless a complete faunal analysis based on extensive exploration is made throughout the whole area of southeastern Iran. 


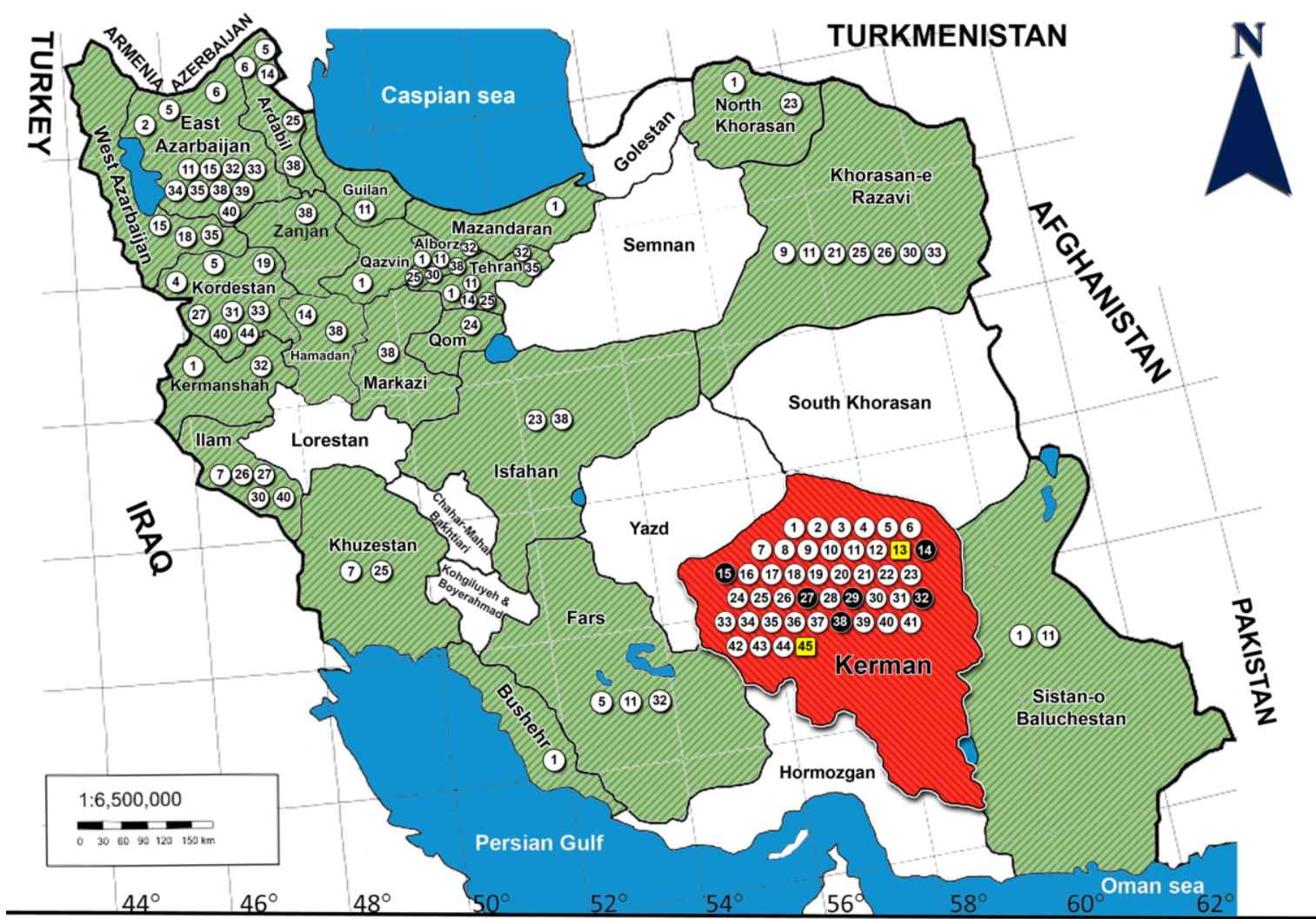

1. Anisopteromalus calandrae (Howard, 1881)

2. Caenacis inflexa (Ratzeburg, 1848)

3. Caenocrepis arenicola (Thomson, 1878)

4. Callitula bicolor Spinola, 1811

5. Catolaccus crassiceps (Masi, 1911)

6. Cheiropachus quadrum (Fabricius, 1787)

7. Chlorocytus spicatus (Walker, 1835)

8. Coelopisthia areolata Askew, 1980

9. Cyrtoptyx latipes (Rondani, 1874)

10. Dibrachys lignicola Graham, 1969

11. Dibrachys microgastri (Bouché, 1834)

12. Dinarmus acutus (Thomson, 1878)

13. Dinarmus altifrons (Walker, 1862)

14. Euneura lachni (Ashmead, 1887)

15. Habritys brevicornis (Ratzeburg, 1844)

16. Hobbya stenonota (Ratzeburg, 1848)

17. Homoporus apharetus (Walker, 1839)

18. Homoporus febriculosus (Girault, 1917)

19. Homoporus fulviventris (Walker 1835)

20. Mesopolobus fasciiventris Westwood, 1833

21. Mesopolobus sericeus (Forster, 1770)

22. Norbanus brevicornis Szelenyi, 1974

23. Novitzkyanus cryptogaster Bouček, 1961
24. Pachycrepoideus vindemmiae (Rondani, 1875)

25. Pachyneuron aphidis (Bouché, 1834)

26. Pachyneuron erzurumicum Doğanlar, 1986

27. Pachyneuron formosum Walker, 1833

28. Pachyneuron gibbiscuta Thomson, 1878

29. Pachyneuron grande Thomson, 1878

30. Pachyneuron groenlandicum (Holmgren, 1872)

31. Pachyneuron leucopiscida Mani, 1939

32. Pachyneuron muscarum (Linnaeus, 1758)

33. Pachyneuron nelsoni Girault, 1928

34. Peridesmia discus (Walker, 1835)

35. Pteromalus bedeguaris (Thomson, 1878)

36. Pteromalus cyniphidis (Linnaeus, 1758)

37. Pteromalus dolichurus (Thomson, 1878)

38. Rhaphitelus maculatus Walker, 1834

39. Sphegigaster ineus Mitroiu, 2008

40. Sphegigaster nigricornis (Nees, 1834)

41. Sphegigaster pedunculiventris (Spinola, 1808)

42. Sphegigaster persiana Mitroiu \& Madjdzadeh, 2011

43. Sphegigaster truncata Thomson, 1878

44. Stenoselma nigrum Delucchi, 1956

45. Syntomopus incurvus Walker, 1833

Figure 5. Distribution map for species of Pteromalinae in Kerman province. Number in black and yellow shapes indicating newly recorded species from Kerman and Iran, respectively. 
Table 1. List of the known Pteromalinae species in Kerman province.

\begin{tabular}{|c|c|c|}
\hline & Species name & References \\
\hline 1 & Anisopteromalus calandrae (Howard, 1881) & Shojaey et al. (2021) \\
\hline 2 & Caenacis inflexa (Ratzeburg, 1848) & Mahdavi et al. (2015) \\
\hline 3 & Caenocrepis arenicola (Thomson, 1878) & Shojaey et al. $(2019,2021)$, current study \\
\hline 4 & Callitula bicolor Spinola, 1811 & Shojaey et al. (2021) \\
\hline 5 & Catolaccus crassiceps (Masi, 1911) & Shojaey et al. (2021) \\
\hline 6 & Cheiropachus quadrum (Fabricius, 1787) & Mitroiu et al. (2011); current study \\
\hline 7 & Chlorocytus spicatus (Walker, 1835) & Shojaey et al. (2021) \\
\hline 8 & Coelopisthia areolata Askew, 1980 & Shojaey et al. (2021) \\
\hline 9 & Cyrtoptyx latipes (Rondani, 1874) & Mitroiu et al. (2011), Shojaey et al. (2021) \\
\hline 10 & Dibrachys lignicola Graham, 1969 & Ziaaddini et al. (2014) \\
\hline 11 & Dibrachys microgastri (Bouché, 1834) & Mitroiu et al. (2011) \\
\hline 12 & Dinarmus acutus (Thomson, 1878) & Mitroiu et al. (2011) \\
\hline 13 & Dinarmus altifrons (Walker, 1862) & Current study \\
\hline 14 & Euneura lachni (Ashmead, 1887) & Current study \\
\hline 15 & Habritys brevicornis (Ratzeburg, 1844) & Current study \\
\hline 16 & Hobbya stenonota (Ratzeburg, 1848) & Lotfalizadeh et al. (2012) \\
\hline 17 & Homoporus apharetus (Walker, 1839) & Shojaey et al. (2021), current study \\
\hline 18 & Homoporus febriculosus (Girault, 1917) & Shojaey et al. (2021) \\
\hline 19 & Homoporus fulviventris (Walker 1835) & Mitroiu et al. (2011), Shojaey et al. (2021) \\
\hline 20 & Mesopolobus fasciiventris Westwood, 1833 & Mahdavi \& Madjdzadeh (2013) \\
\hline 21 & Mesopolobus sericeus (Förster, 1770) & Mahdavi et al. (2015) \\
\hline 22 & Norbanus brevicornis Szelenyi, 1974 & Shojaey et al. (2021) \\
\hline 23 & Novitzkyanus cryptogaster Bouček, 1961 & Rahmani et al. (2019) \\
\hline 24 & Pachycrepoideus vindemmiae (Rondani, 1875) & Shojaey et al. (2021), current study \\
\hline 25 & Pachyneuron aphidis (Bouché, 1834) & $\begin{array}{l}\text { Mitroiu et al. (2011), Shojaey et al. (2021), } \\
\text { current study }\end{array}$ \\
\hline 26 & Pachyneuron erzurumicum Doğanlar, 1986 & Mitroiu et al. (2011), current study \\
\hline 27 & Pachyneuron formosum Walker, 1833 & Current study \\
\hline 28 & Pachyneuron gibbiscuta Thomson, 1878 & Shojaey et al. (2021) \\
\hline 29 & Pachyneuron grande Thomson, 1878 & Current study \\
\hline 30 & Pachyneuron groenlandicum (Holmgren, 1872) & Mitroiu et al. (2011), current study \\
\hline 31 & Pachyneuron leucopiscida Mani, 1939 & Shojaey et al. (2021) \\
\hline 32 & Pachyneuron muscarum (Linnaeus, 1758) & Current study \\
\hline 33 & Pachyneuron nelsoni Girault, 1928 & Shojaey et al. (2021), current study \\
\hline 34 & Peridesmia discus (Walker, 1835) & Shojaey et al. (2021) \\
\hline 35 & Pteromalus bedeguaris (Thomson, 1878) & Lotfalizadeh et al. (2012) \\
\hline 36 & Pteromalus cyniphidis (Linnaeus, 1758) & Mahdavi et al. (2015) \\
\hline 37 & Pteromalus dolichurus (Thomson, 1878) & Mahdavi et al. (2015) \\
\hline 38 & Rhaphitelus maculatus Walker, 1834 & Current study \\
\hline 39 & Sphegigaster ineus Mitroiu, 2008 & Shojaey et al. (2021) \\
\hline 40 & Sphegigaster nigricornis (Nees, 1834) & Shojaey et al. (2021) \\
\hline 41 & Sphegigaster pedunculiventris (Spinola, 1808) & Shojaey et al. (2021) \\
\hline 42 & Sphegigaster persiana Mitroiu \& Madjdzadeh, 2011 & Mitroiu et al. (2011) \\
\hline 43 & Sphegigaster truncata Thomson, 1878 & Mitroiu et al. (2011) \\
\hline 44 & Stenoselma nigrum Delucchi, 1956 & Shojaey et al. (2021) \\
\hline 45 & Syntomopus incurvus Walker, 1833 & Current study \\
\hline
\end{tabular}


Table 2. Biogeographical distribution of the Pteromalinae species in Kerman province.

\begin{tabular}{|l|l|l|}
\hline Distribution & No of species & Frequency (\%) \\
\hline Palaearctic & 19 & 42.2 \\
\hline Holarctic (Palaearctic and Nearctic) & 9 & 20 \\
\hline Palaearctic and Oriental & 3 & 6.7 \\
\hline Palaearctic, Afrotropical and Oriental & 3 & 6.7 \\
\hline Palaearctic, Nearctic and Oriental & 1 & 2.2 \\
\hline
\end{tabular}

Acknowledgments - This study was supported by grant No. UOZ-GR-9618-6, University of Zabol. The authors also thank Sh. Mohebban, M. Azad-Biglari, and N. Amirinasab, for collecting the core material for this study.

\section{REFERENCES}

Abolhassanzadeh, F., Lotfalizadeh, H. \& MADJDZADEH, S.M. (2017): Updated checklist of Pteromalidae (Hymenoptera: Chalcidoidea) of Iran, with some new records. Journal of Insect Biodiversity and Systematics, 3(2): 119-140.

BASIRI, N., LOTfalizadeH, H. \& KAZEMI, M.H. (2012): Introduction to parasitoids of bark beetles (Coleoptera: Scolytidae) in Meshkin-Shahr, Ardebil province, Iran. Journal of Field Crop Entomology, 2(1): 53-64.

BOUČEK, Z. \& RASPLUS, J.Y. (1991): Illustrated key to West-Palearctic genera of Pteromalidae (Hymenoptera: Chalcidoidea). Institut National de la Recherche Agronomique, Paris, 140 pp.

DehDAR, K. \& MAdJdZAdeH, S.M. (2016): Pteromalidae (Hymenoptera: Chalcidoidea) from Kordestan province, western Iran. Far Eastern Entomologist, (315): 11-20.

DZHANOKMEN, K.A. (2009): Review of Kazakhstan species of the genus Pachyneuron Walker, 1833 (Hymenoptera: Chalcidoidea: Pteromalidae). Zoosystematica Rossica, 18(1): 73-82. doi: $\underline{10.31610 / \mathrm{zsr} / 2009.18 .1 .73}$

EBRAHIMI, E. (2014): Parasitoid and hyperparasitoid wasps of scale insects in Hayk Mirzayans Insect Museum, Iran. Journal of Entomological Society of Iran, 34(1): 73-83.

ESLAMI, J. (1998): The proof of larval gregarism in Dinarmus vagabundus (Hym.: Pteromalidae) and a study of some of its population problems. Proceeding of the $13^{\text {th }}$ Iranian Plant Protection Congress, 23-27August, Karaj, Iran, p. 70.
FARAhani, H.K., Goldansaz, S.H., SABAhI, G. \& BAUR, H. (2010): First report of Pachycrepoideus vindemmiae (Hym.: Pteromalidae) from Iran. Journal of Entomological Society of Iran, 29(2): 117118.

Gibson, G.A., Huber, J.T. \& Woolley, J.B. (Eds.) 1997: Annotated Keys to the Genera of Nearctic Chalcidoidea (Hymenoptera). NRC Research Press, Ottawa, Ontario, Canada. 794 pp.

Gibson, G.A., Dzhanokmen, K.A., VAn NoOrt, S., GHAHARI, H. \& DOGANLAR, M. (2021): Family Pteromalidae Dalman, 1820. Chalcidoidea of Iran: (Insecta: Hymenoptera). In: GHAHARI, H., GIBSON, G. \& Viggiani, G. (Eds.) Chalcidoidea of Iran:(Insecta: Hymenoptera). CABI. pp. 295-353. doi: $\underline{10.1079 / 9781789248463.0295}$

GraHAM, M.W.R.D.V. (1969): The Pteromalidae of North-Western Europe (Hymenoptera: Chalcidoidea). British Museum, Natural History, 16: 1908. doi: $10.5962 / \mathrm{p} .258046$

HAESElBARTH, E. (1983): Determination list of entomophagous insects. No. 11. Bulletin Section Regionale Ouest Palaearctique Organisation Internationale de Lutte Biologique 12(7): 1-62.

HASANI, A. \& MADJDZADEH, S.M. (2012): Contribution to the knowledge of the Pteromalidae (Hymenoptera: Chalcidoidea) from Khorasan Razavi province, Northeastern Iran. Iranian Journal of Animal Biosystematics, 8 (1): 57-69.

Hasani, A., MitroiU, M.D. \& Madjdzadeh, S.M. (2011): New records of Pteromalidae (Hymenoptera: Chalcidoidea) from Northeastern Iran. Acta Zoologica Bulgarica, 63(3): 323-325.

HASSAN-PASHAIMEHR, M. \& LOTFALIZADEH, $\mathrm{H}$. (2015): Discovery of Habritys brevicornis (Ratzeburg, 1844) (Hymenoptera: Pteromalidae) in the Middle-East. Journal of Insect Biodiversity and Systematics, 1(1): 33-36. 
Jalilvand, K., Shirazi, M., Fallahzadeh, M., VAHEDI, H.A., SAMIH, M.A. \& NAGHADEH, N.M. (2014): Survey of natural enemies of mealybug species (Hemiptera, Pseudococcidae) in Kermanshah province, western Iran to inform biological control research. Journal of the Entomological Research Society, 16(3): 1-10.

Lotfalizadeh, H. \& AHMADI, A. (2000): Natural enemies of cypress tree mealybug Planococcus vovae (Nasonov) and their parasitoids in Shiraz, Iran. Iran Agricultural Research, 19(2): 145-154.

Lotfalizadeh, H. \& Gharali, B. (2008): Pteromalidae (Hymenoptera: Chalcidoidea) of Iran: New records and a preliminary checklist. Entomofauna, 29(6): 93-120.

LOTFALIZADEH, H., RAJABI, M. \& MADJDZADEH, S.M. (2012): Parasitoids community of Diplolepis fructuum (Rübsaamen) (Hym.: Cynipidae) in Kerman province, with checklist of its associated Hymenoptera fauna in Iran. North-Western Journal of Zoology, 8(1): 125-131.

LOTFALIZADEH, H., ZARGARAN, M.R. \& TAGHIZADEH, M. (2014): Species diversity of Coccoidea parasitoids wasps (Hym.: Chalcidoidea) in the northern parts of East-Azarbaijan province, Iran. NorthWestern Journal of Zoology, 10(1): 60-66.

Lotfalizadeh, H., Karimpour, Y., Delvare, G. \& RASPLUS, J.Y. (2020): Chalcidoidea (Hymenoptera) obtained from common reed, Phragmites australis (Cav.) Trin. ex Steud. (Poaceae) in Iran with new records and descriptions of two new species. European Journal of Taxonomy, 710: 135. doi: $10.5852 /$ ejt.2020.710

Mahdavi, M. \& MAdJdzadeH, S.M. (2013): Contribution to the knowledge of Chalcidoidea (Pteromalidae and Eupelmidae) of Iran. North-Western Journal of Zoology, 9(1): 94-98.

MahdaVi, M., MadjDZAdeh, S.M. \& MitroiU, M.D. (2015): Pteromalidae (Hymenoptera: Chalcidoidea) associated with plant galls in the south-eastern Iran, with three new records. Journal of Insect Biodiversity and Systematics, 1(1): 47-54.

MitroiU, M.D. (2012): The genus Caenocrepis Thomson (Hymenoptera: Pteromalidae) in the Afrotropical region, with a key to world species. Zootaxa, 3557(1): 49-55. doi: 10.11646/zootaxa.3557.1.5

Mitroiu, M.D., ABolhassanzadeh, F. \& MADJDZADEH, S.M. (2011): New records of Pteroma- lidae (Hymenoptera: Chalcidoidea) from Iran, with description of a new species. North Western Journal of Zoology, 7(2): 243-249.

Moravvej, S.A., LotFalizadeH, H. \& SHISHEHBor, P. (2018): A contribution to the study of Pteromalidae (Hymenoptera: Chalcidoidea) of Khuzestan in southwestern Iran. Journal of Insect Biodivesity and Systematics, 4(2): 91-97.

NoYeS, J.S. (2019): Universal Chalcidoidea Database. World Wide Web electronic publication. http://www.nhm.ac.uk/chalcidoids (accessed 21 June, 2021).

OILB (1971): Liste d'identification des entomophages. Organisation Internationale de Lutte Biologique (OILB), Genève, 8: 1-18.

RAHMANI, Z., RAKHSHANI, E., LOTFALIZADEH, H. \& MOKHTARI, A. (2019): Two small genera, Ischyroptyx Delucchi and Novitzkyanus Bouček (Hymenoptera: Pteromalidae) new to fauna of Iran. Oriental Insects, 54(4): 564-573. doi: $\underline{10.1080 / 00305316.2019 .1697767}$

RAHMANI, Z., RAKHSHANI, E., LOTFALIZADEH, H. \& MOKHTARI, A. (2020): First records of the genera Psilocera Walker, 1833 and Stinoplus Thomson, 1878 (Hymenoptera, Pteromalidae) in Iran. Journal of Insect Biodiversity and Systematics, 6(3): 213221.

Rakhshani, E., TAleBI, A.A., SAdeghi, E., KaVAlliERATOS, N.G. \& RASHED, A. (2004): Seasonal parasitism and hyperparasitism of valnus aphid, Chromaphis juglandicola (Hom.: Aphididae) in Tehran province. Journal of Entomological Society of Iran, 23(2): 1-11.

RaKhshani, E., TAlebi, A., StarÝ, P., MANZARI, S. \& REZWANI, A. (2005): Re-description and biocontrol information of Pauesia antennata (Mukerji) (Hym., Braconidae, Aphidiinae), parasitoid of Pterochloroides persicae (Chol.) (Hom., Aphidoidea, Lachnidae). Journal of Entomological Research Society, 7(3): 59-59.

RASPLUS, J.Y. (1989): Révision des espèces afrotropicales du genre Dinarmus Thomson (Hymenoptera: Pteromalidae). Annales de la Société Entomologique de France, 25(2): 135-162.

SADEGHI, S.E. \& EBRAHIMI, E. (2001): New report of Pachyneuron grande Thomson (Hym.: Pteromalidae) from Iran. Journal of Entomological Society of Iran, 21(1): 113-114. 
Shojaey, M., KhaYrandish, M., Madjdzadeh, S.M. \& LOTFALIZADEH, H. (2019): First record of Caenocrepis arenicola (Thomson, 1878) (Hymenoptera: Pteromalidae) from Iran. Journal of Insect Biodiversity and Systematics, 5(2): 121-126.

ShOJAEY, M., KHAYRANDISH, M., MADJDZADEH, S.M. \& LotfalizadeH, H. (2021): New records of Pteromalinae (Hymenoptera: Chalcidoidea, Pteromalidae) from Iran. Far Eastern Entomologist, 439: 14-23. doi: $\underline{10.25221 / \text { fee. } 439.2}$

SURESHAN, P.M. \& NARENDRAN, T.C. (2001): Taxonomic studies on Dinarmus Thomson (Hymenoptera:
Chalcidoidea: Pteromalidae) of India and adjacent countries. Zoos'print Journal, 16(12): 643-650. doi: 10.11609/JoTT.ZPJ.16.12.643-50

VAN ACHTERBERG, C. (2009): Can Townes type Malaise traps be improved? Some recent developments. Entomologische Berichten, 69: 129135.

ZIAADDINI, M., LOTFALIZADEH, H., MOHAMMADI KhORAMABADI, A. \& JALALI, M.A. (2014): First report of Dibrachys lignicola (Hym.: Pteromalidae) from Iran. Journal of Entomological Society of Iran, 34(1): 97-98. 